\title{
A Novel Approach For Texture Shape Recovery
}

\author{
Jing Wang \\ Kristin J. Dana \\ Electrical and Computer Engineering Department \\ Rutgers University \\ Piscataway, New Jersey 08854 \\ jingwang,kdana@caip.rutgers.edu
}

\begin{abstract}
In vision and graphics, there is a sustained interest in capturing accurate $3 D$ shape with various scanning devices. However, the resulting geometric representation is only part of the story. Surface texture of real objects is also an important component of the representation and finescale surface geometry such as surface markings, roughness, and imprints, are essential in highly realistic rendering and accurate prediction. We present a novel approach for measuring the fine-scale surface shape of specular surfaces using a curved mirror to view multiple angles in a single image. A distinguishing aspect of our method is that it is designed for specular surfaces, unlike many methods (e.g. laser scanning) which cannot handle highly specular objects. Also, the spatial resolution is very high so that it can resolve very small surface details that are beyond the resolution of standard devices. Furthermore, our approach incorporates the simultaneous use of a bidirectional texture measurement method, so that spatially varying bidirectional reflectance is measured at the same time as surface shape.
\end{abstract}

\section{Introduction}

Capturing high-quality digital models is a central issue for research in computer vision and graphics. With ubiquitous use of the internet, there are an increasing number of applications for such models such as e-commerce, historical archiving, and virtual reality. Acquiring depth maps with three-dimensional scanning is a main component of systems that capture digital object models. There are several methods for range scanning or 3D scanning which are based on time of flight, depth from defocus, and projectlight triangulations. Typical methods of 3D scanning cannot fully represent the appearance of an object. There are three main problems and each of these are solved with the approach described here.
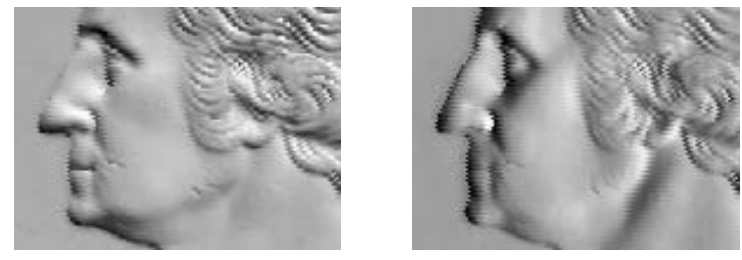

Figure 1. An illustration of the estimated surface normals for a coin surface. The rendering shown is constructed by applying the lambertian shading model to the estimated surface normals, with an illumination polar angle of $30^{\circ}$ and an azimuth of $60^{\circ}$ (left), $0^{\circ}$ (right). Notice that fine-scale structural details are preserved including small scratches by the mouth and neck. The presence and appearance of these scratches are visually consistent with observations of the coin.

The first problem in using geometry from 3D scanning for object modeling is that depth information alone is not a sufficient representation of an object. Reflectance is an equally important property and the naive approach of combining geometry with simple reflectance models yields an inaccurate object representation. The typical approach to solve this problem uses two devices (e.g. camera and range scanner as in [18]) so that both images and geometry can be captured. However because multiple devices are used, there is an underlying registration problem. Also, a single image cannot capture the variation of surface appearance with camera and illumination direction. Studies of the bidirectional texture function (BTF) including the work of [10][2][15][17][19][5][12] show the importance of representing the variation of local appearance with imaging parameters. Many modern approaches for digital model acquisition such as [6][13] are image-based in order to capture surface detail. Bidirectional imaging is required for a complete object representation, but it's difficult to do bidirectional imaging and shape capture simultaneously. Yet this is 
exactly what our approach accomplishes. The bidirectional texture function (BTF) is measured with exactly the same apparatus that measures surface shape. There is no need to employ multiple cameras or multiple light sources. This novel approach eliminates the need for any post-processing registration step and therefore greatly simplifies the capture process while increasing overall accuracy.

The second difficulty with 3D scanning methods is the limited depth and spatial resolution that obstructs capturing fine-scale surface geometry. Studies of the appearance of real world surfaces including [4][9][8] underscore the importance of accounting for fine-scale surface texture such as roughness, fur, pits, grooves, etc. Our approach captures fine-scale geometry in a high level of detail. Figure 1 shows a rendering of a coin that was obtained using a surface normal map estimated with our method. The figure reveals the high quality depth resolution that is achieved. The overall fine geometry is clearly recovered in detail. Even the small surface scratches around the mouth and neck region are clearly visible and are consistent with visual observations of this coin. High resolution methods in the state of the art of 3D scanning include [1], [11], and [16]. In [1], the range images are captured by a multi-camera stereo system which projects a striped pattern onto the target object, and achieves an intersample distance of $2 \mathrm{~mm}$ with a submillimeter depth accuracy. In [11], laser-stripe triangulation is used with a sample distance of $1 / 4 \mathrm{~mm}$ along the laser stripe and depth resolution at least twice this fine. In [16], projected structured-light triangulation is used for range scanning with the sample spacing $0.5-0.75 \mathrm{~mm}$. Our method achieves high spatial resolution since the sample spacing depends mostly on the stage increments and these are quite small. In Figure 1, the horizontal and vertical intersample spacing is approximately $0.1 \mathrm{~mm}$ and clearly the depth accuracy is submillimeter.

A third common problem that is encountered when building object models is that current scanning methods work well for matte objects but work poorly for specular objects. There are several reasons that specular objects are problematic. First of all, in light projection techniques, the pattern is expected to be affected by object shape and the change in the pattern due to a specular highlight introduces errors. Also, methods that use multiple devices or cameras have a post registration step. Image registration is based on brightness constancy and is notoriously vulnerable to errors in the presence of specularities because they violate the brightness constancy assumption. Our approach obtains shape information from the specularity and therefore is ideal for the complementary group of objects that is not well captured by existing devices. While existing methods work best for matte objects, our method works best for specular or partially specular objects.

The method we present here directly calculates the sur- face normal map from multi-view images. Multiview images are images of a surface point observed over a range of viewing angles. Normally such images are difficult to obtain, but by employing the bidirectional imaging device described in [3] we can instantaneously obtain the multiview observation of a point. Multiview images are obtained by scanning the surface point-by-point in the scan-line order. The spatial pixel size is controlled by the scanning step and not the CCD array, and we can achieve a spatial resolution less than $0.1 \mathrm{~mm}$. There is no need for registration of geometry and images and there is no need for computing point correspondence as in stereo-based methods. The point that is imaged is exactly the same point where the surface normal is measured. Therefore our method can capture the bidirectional reflectance of the surface point and the surface normal of that same surface point simultaneously. Through scanning, the bidirectional texture function and the surface normal map is obtained. Previous work in shape from specularity such as [7] [14] uses photometric sampling and requires either multiple light sources, a moving light source or an extended light source. Our approach is fundamentally different because the curved mirror enables multiple viewing directions simultaneously. So the specularity is visible using a single light direction and the location of the specularity reveals the local surface normal.

\section{Device Design}

The device described in [3] has been adapted in this work to provide a measurement of shape as well as image texture. We summarize the main imaging components for this device. A concave parabolic mirror has the useful optical property that light parallel to the mirror axis is focused to a single point. This property enables control of illumination angle, by simply moving the incident illumination ray to a different part of the mirror. Additionally all light reflected from the surface point is collected by the mirror and diverted to a set of parallel reflected rays. As described in [3], the device uses the concave parabolic mirror along with a beam-splitter, CCD camera, and translation stages and is illustrated in Figure 2. The beam-splitter allows simultaneous control of the illumination and viewing direction. A concave parabolic mirror section is positioned so that its focus is coincident with the surface point to be measured. The illumination source is a collimated beam of light parallel to the global plane of the surface and passing through a movable aperture. The aperture ensures that only a spot of the concave mirror is illuminated and therefore one illumination direction is measured for each aperture position. In this approach, the problem of changing illumination direction over a hemisphere is transformed to the easier problem of translating an aperture in a plane. The light reflected at each angle is reflected from the mirror to a parallel direction and 

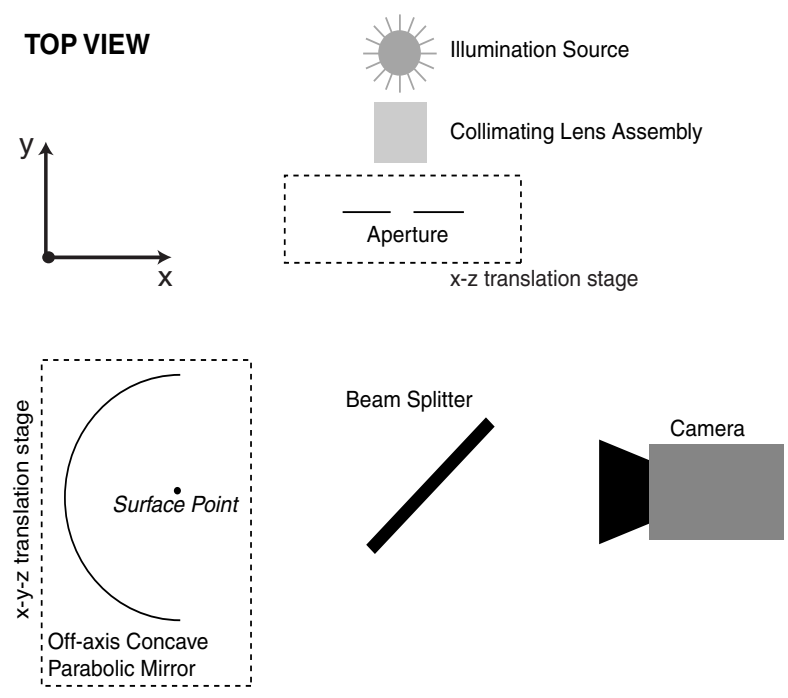

Figure 2. Texture camera (texcam). The surface point is imaged by a CCD video camera observing an off-axis concave parabolic mirror for simultaneous observation of a large range of viewing directions. Illumination direction is controlled by an aperture, i.e. translations of the aperture in the $\mathrm{x}-\mathrm{z}$ plane cause variations in the illumination angle incident on the surface point. The device achieves illumination/viewing direction variations using a simple translations of the illumination aperture instead of complex gonioreflectometer equipment. Measurements of bidirectional texture are accomplished by translating the mirror in the $x-y$ plane. The position of the specularity is detected in the observed camera images and used to estimate the surface normal.

diverted by the beam-splitter to the camera. The camera is equipped with an orthographic lens that images the parallel light directly incident on the lens. In this manner, a single image corresponds to reflectance measurements from all angles in a partial hemisphere. To obtain a measurement of a surface patch for spatially varying BRDF (i.e. BTF), the concave mirror is translated along the $X-Y$ plane. Note that the range of viewing and illumination angles can be increased by increasing the length of the parabolic mirror section. Also for shapes that are not planar, an $X-Y-Z$ translation stage can be used to support the concave mirror and enable scanning along the surface shape.

The equation for the surface of the parabolic mirror as shown in Figure 3 is

$$
y+F=\frac{z^{2}+x^{2}}{4 F},
$$

where $F$ is parent focal length $(12.7 \mathrm{~mm}$ for the current prototype). This function is obtained by rotating the parabolic curve $y+F=\frac{z^{2}}{4 F}$ about the $Y$ axis. The im-

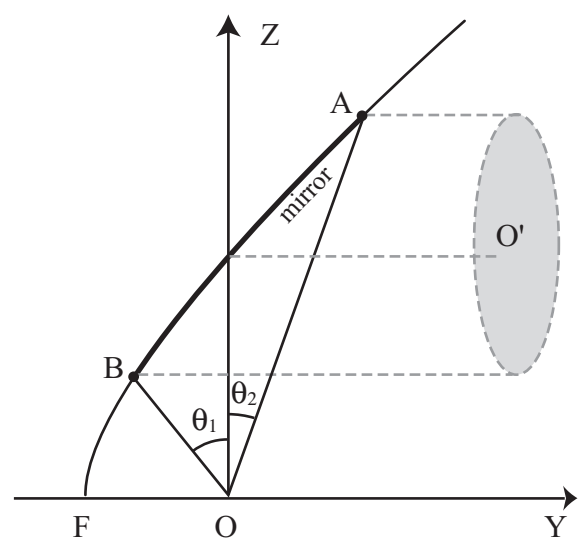

Figure 3. The cross-section of the concave parabolic mirror used in the texture camera (texcam). The off-axis mirror section extends from point $A$ to point $B$. Light parallel to the mirror axis, i.e. the $Y$ axis, is focused to point $\mathrm{O}$.

age of the mirror is viewed by the camera (with telecentric lens) that is positioned so that its optical axis lies along the $Y$ axis. The point on the mirror with coordinates $x=0$, $y=0, z=2 F=25.4 \mathrm{~mm}$ is the point in the center of the projected image. Since the radius of the projected image is $12.7 \mathrm{~mm}$, we can easily calculate the coordinates of $B$ in Figure 3 as

$$
\begin{aligned}
& z_{B}=12.7=F \\
& y_{B}=\frac{z_{B}^{2}+x_{B}^{2}}{4 F}-F=-\frac{3}{4} F .
\end{aligned}
$$

The angle $\theta_{1}$ is

$$
\theta_{1}=\arctan \left(\frac{3}{4}\right)=36.8^{\circ}
$$

By the same method, the coordinates of $A$ in Figure 3 are expressed as

$$
\begin{aligned}
& z_{A}=3 \times 12.7=3 F, \\
& y_{A}=\frac{z_{A}^{2}+x_{A}^{2}}{4 F}-F=\frac{5}{4} F .
\end{aligned}
$$

The angle $\theta_{2}$ is

$$
\theta_{2}=\arctan \left(\frac{5}{12}\right)=22.6^{\circ}
$$

Since $\theta_{1}$ and $\theta_{2}$ are less than $90^{\circ}$, all angles of the hemisphere are not imaged with the current texcam prototype. By increasing the size of the parabolic mirror section both 


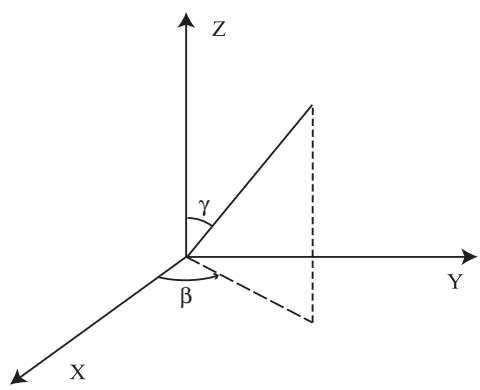

Figure 4. Definition of relevant angles for the analysis of specular reflection. The polar angle of the illumination is $\gamma$, and the azimuthal angle is $\beta$.

$\theta_{1}$ and $\theta_{2}$ can approach $90^{\circ}$. However, while $\theta_{1}$ can be increased easily, increases in $\theta_{2}$ cause a large increase in the length of the mirror section.

While this device was originally intended for capturing reflectance and texture, we now consider an adaptation for getting shape from specularity. The camera image is a circle of $25.4 \mathrm{~mm}$ diameter and is the view looking down the $Y$ axis. Each pixel of the camera image corresponds to a viewing direction, and so the camera image is a multiview image of a single surface point. With proper positioning of the illumination, a specularity can be detected. Since the illumination and viewing direction are known, the position of this specularity reveals the surface normal.

\section{Specular Surfaces}

Consider the image formation process when using this device to image a specular surface. When imaging such a surface, we expect a component of specular reflection as shown in Figure 6. Assume a specular sample is at the focus of the mirror with its global surface normal aligned with the $Z$ axis. The intersection of the illuminating ray and the mirror is at position $P$ given by coordinates $(x, y, z)$. Figure 4 illustrates relevant angles. The polar angle is given by

$$
\gamma=\arccos \frac{z}{\sqrt{x^{2}+y^{2}+z^{2}}}
$$

and the azimuth angle $\beta$ expressed as

$$
\beta=\arccos \frac{x}{\sqrt{x^{2}+y^{2}}} .
$$

For a specularity, the reflected ray has the same polar angle as the incident ray, while the azimuth angle is $\beta-\pi$. To predict where the specularity will be for a given aperture position, (or equivalently, a given mirror intersection $P$ ) consider how $\gamma$ and $\beta$ are related to the $x, y, z$, the coordinates of $P$. The equations for $\beta$ and $\gamma$ are

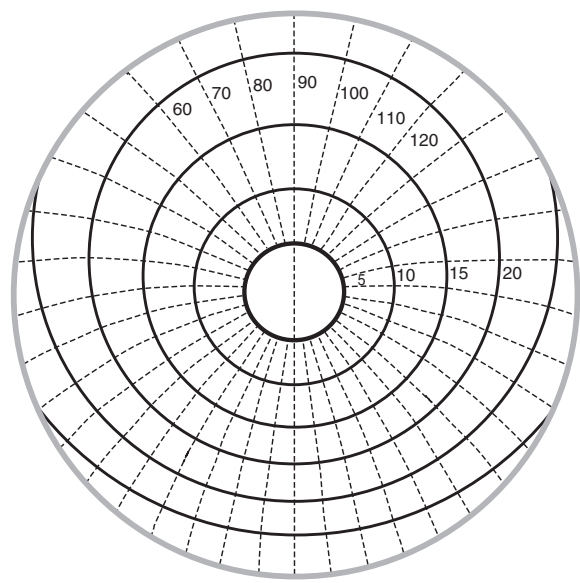

Figure 5. Curves of constant $\gamma$ and constant $\beta$. The gray bold circle represents the size of the projected image. Each black ellipse is curves of constant $\gamma$. (Shown from inner most to the outer most with step size $5^{\circ}, \gamma=$ $5,10, \ldots .25$.) The dashed radiating lines are curves of constant $\beta$. (Shown increasing clockwise with a step size of $\left.10^{\circ}, \beta=60,70, \ldots\right)$

$$
\begin{aligned}
& \cos \beta=\frac{x}{\sqrt{x^{2}+y^{2}}}, \\
& \sin \beta=\frac{y}{\sqrt{x^{2}+y^{2}}}, \\
& \sin \gamma=\frac{\sqrt{x^{2}+y^{2}}}{\sqrt{x^{2}+y^{2}+z^{2}}} .
\end{aligned}
$$

Using Equation 1,

$$
\begin{aligned}
\sin \gamma & =\frac{\sqrt{x^{2}+y^{2}}}{\sqrt{y^{2}+4 F y+4 F^{2}}}=\frac{\sqrt{x^{2}+y^{2}}}{|y+2 F|} \\
& =\frac{|y|}{|y+2 F|} \sqrt{\left(\frac{x}{y}\right)^{2}+1}=\frac{|y|}{|y+2 F|} \sqrt{\left(\frac{\cos \beta}{\sin \beta}\right)^{2}+1} \\
& =\frac{|y|}{|y+2 F||\sin \beta|} .
\end{aligned}
$$

Since $y+F=\frac{z^{2}+x^{2}}{4 F}>0, y+2 F>0$. The equation becomes

$$
\sin \gamma|\sin \beta|=\frac{|y|}{y+2 F} .
$$

We now need to determine the sign of $y$. This sign is the same as that of $\sin \beta$, so we can get rid of the absolute value function as

$$
\sin \gamma \sin \beta=\frac{y}{y+2 F},
$$




$$
y=\frac{2 F}{\frac{1}{\sin \gamma \sin \beta}-1},
$$

and

$$
\begin{aligned}
& x=\frac{y}{\tan \beta}, \\
& z=\sqrt{4 F(y+F)-x^{2}} .
\end{aligned}
$$

Using these relationships we can plot lines of constant $\gamma$ and $\beta$ on the camera image as shown in Figure 5.

\section{Shape Recovery}

The imaging device can be used for shape recovery by first detecting the position of the specularity for a given aperture position. The specularity is found in the camera image, such as that shown in Figure 6. First, we find the positions of the largest intensity value and then the centroid of these positions is computed. (To increase the accuracy of this step, high dynamic range imaging can be employed.)

The transformation from the coordinates shown in Figure 3 to the screen coordinates $x_{s}, y_{s}$ is straightforward,

$$
\begin{aligned}
& x_{s}=-x / p+C_{x}, \\
& y_{s}=-(z-2 F) / p+C_{y},
\end{aligned}
$$

where $C x$ and $C_{y}$ are the center of the screen, for the $640 \times 480$ setting, $C x=319, C_{y}=239$.

By using the Equation 17, we can convert to the coordinates shown in Figure 3 from the screen coordinates of the detected specularity. Next, the polar and azimuth angle of the specular reflection direction is computed using Equations 6 and 7 .

Suppose the incident light has the direction $\gamma_{L}$ and $\beta_{L}$, and the specular reflection of the sample surface has the direction $\gamma_{S}$ and $\beta_{S}$. The illumination vector $V_{l}$ is given by

$$
V_{l}=\left(\sin \left(\gamma_{L}\right) \cos \left(\beta_{L}\right), \sin \left(\gamma_{L}\right) \sin \left(\beta_{L}\right), \cos \left(\gamma_{L}\right)\right),
$$

and the specular reflection vector $V_{s}$ is given by

$$
V_{s}=\left(\sin \left(\gamma_{S}\right) \cos \left(\beta_{S}\right), \sin \left(\gamma_{S}\right) \sin \left(\beta_{S}\right), \cos \left(\gamma_{S}\right)\right) .
$$

So, the vector of normal of the surface is:

$$
V_{n}=V_{l}+V_{s}
$$

From this vector, we can easily calculate the surface normal angles $\gamma_{N}$ and $\beta_{N}$.

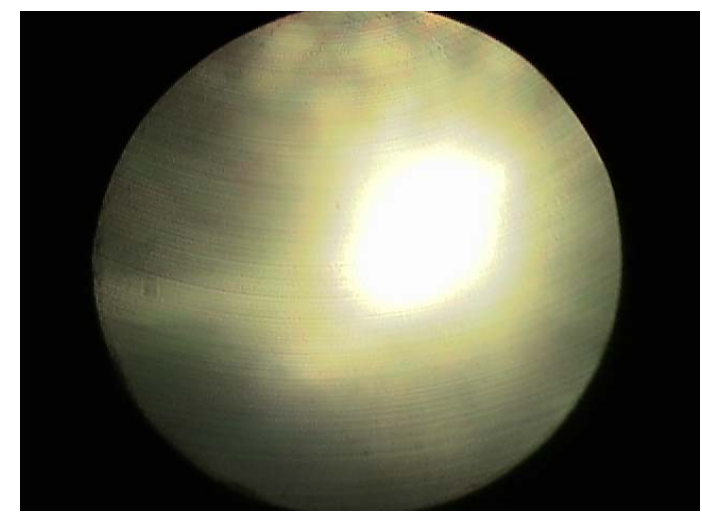

Figure 6. Camera image of the coin using the device shown in Figure 2. Notice the strong specular reflection in a small region. The entire image size is 640 by 480 pixels and the circle region is $25.4 \mathrm{~mm}$ diameter.

\section{Results}

For an initial test of the shape recovery, we determined the surface tilt of ramp objects with known surface tilt. Figure 7 shows the resulting surface tilts (actual and estimated). From this figure we see that the accuracy of the device is quite reasonable. Some sources of error that can be identified are non-ideal optical behavior of the collimated light source, the telecentric lens and the beam-splitter. In addition, optical misalignment of the components can lead to errors.

For the main result, the bidirectional texture and the finescale surface shape is measured for a coin. The shape measurement of the coin is shown in two ways. The actual surface normal estimate is shown in Figure 8 where the $z$ component is displayed as a mesh. Figure 9 shows an additional illustration of the recovered surface shape. Here the object is rendered using a simple lambertian shading model. Given the light direction angles $\gamma_{L}$ and $\beta_{L}$, the lambertian surface rendering equation yields the intensity $I$,

$$
\begin{aligned}
& I=\cos \left(\gamma_{N}\right) \cos \left(\gamma_{L}\right)+ \\
& \quad \sin \left(\gamma_{N}\right) \cos \left(\beta_{N}\right) \sin \left(\gamma_{L}\right) \cos \left(\beta_{L}\right) \\
& \quad+\sin \left(\gamma_{N}\right) \sin \left(\beta_{N}\right) \sin \left(\gamma_{L}\right) \sin \left(\beta_{L}\right)
\end{aligned}
$$

Figure 9 shows the surface normal map rendered as an image using lambertian shading and three different illumination directions.

The bidirectional texture function measured for the coin sample is illustrated in Figure 10 for a sampling of imaging parameters. Notice that this measured texture reveals a very realistic metallic reflectance that has some spatial variation due to the coin imperfections. 


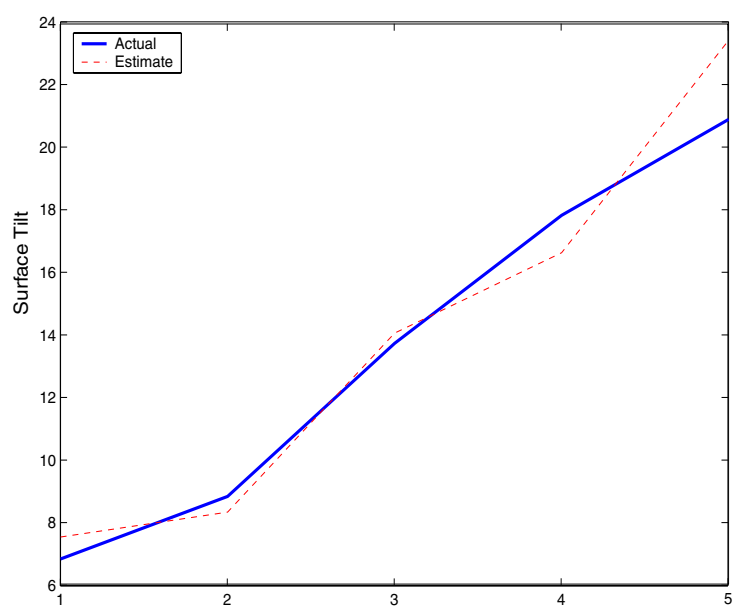

Figure 7. Tilt measurement for a ramp object. Five known surface slants were used. The estimated and actual polar angles of the ramps is plotted for the five cases and for each case the estimated angle is close to the ground truth.

\section{Conclusion}

In summary, we present a new method for the recovery of texture and fine-scale shape. In comparing with other methods, the main advantages of our method are as follows: (1) bidirectional texture and fine-scale surface shape are recovered simultaneously by the same device, (2) the recovered surface shape is very high resolution, (3) specular surfaces can be scanned without the errors present in other range scanning devices. We demonstrate accurate results on a known test object. In addition a coin that has a complex fine-scale surface structure is captured in striking detail. Future work will include evaluating the performance of this method on weakly specular surfaces.

\section{References}

[1] F. Bernardini, I. Martin, and H. Rushmeier. Highquality texture reconstruction from multiple scans. IEEE Transactions on Vision and Computer Graphics, 7(4):318-332, Oct.-Dec 2001.

[2] O. G. Cula and K. J. Dana. Compact representation of bidirectional texture functions. Proceedings of the IEEE Conference on Computer Vision and Pattern Recognition, 1:1041-1067, December 2001.

[3] K. J. Dana. Brdf/btf measurement device. International Conference on Computer Vision, 2:460-6, July 2001.

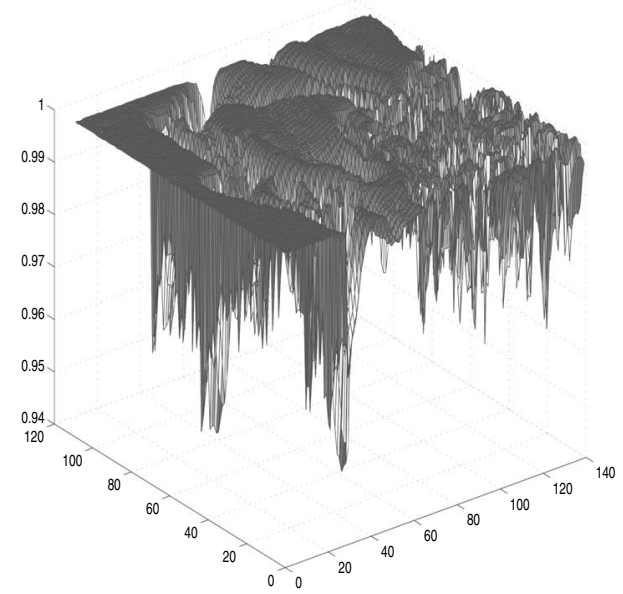

Figure 8. The $z$ component of the surface normals for the coin. Notice the high frequency variation shown around the hairline, the more structured variation around the facial features and the flat middle region corresponding to the cheek area.
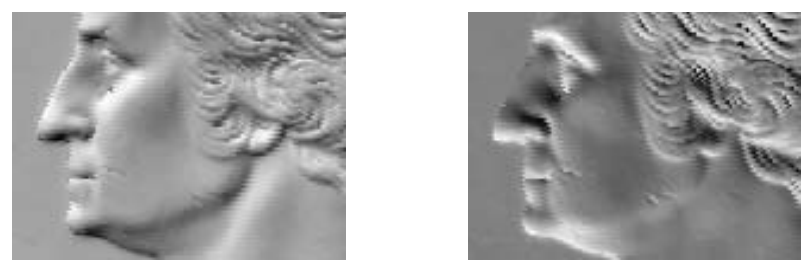

Figure 9. The coin image reconstructed from the lambertian surface assumption applied to the estimated surface normals. From left to right, $\beta_{L}=120^{\circ}, 240^{\circ}$ and for each $\gamma_{L}=45^{\circ}$.

[4] K. J. Dana, B. van Ginneken, S. K. Nayar, and J. J. Koenderink. Reflectance and texture of real world surfaces. ACM Transactions on Graphics, 18(1):1-34, January 1999.

[5] J. Dong and M. Chantler. Capture and synthesis of $3 \mathrm{~d}$ surface texture. Texture2002, The 2nd International Workshop on Texture Analysis andSynthesis, Copenhagen, pages 41-45, June 12002.

[6] T. Hawkins, J. Cohen, and P. Debevec. A photometric approach to digitizing cultural artifacts. 2nd International Symposium on Virtual Reality, Archaeology, and Cultural Her-itage, pages 333-342, 2001.

[7] K. Ikeuchi. Determining surface orientations of specular surfaces by using the photometric stereo method. 

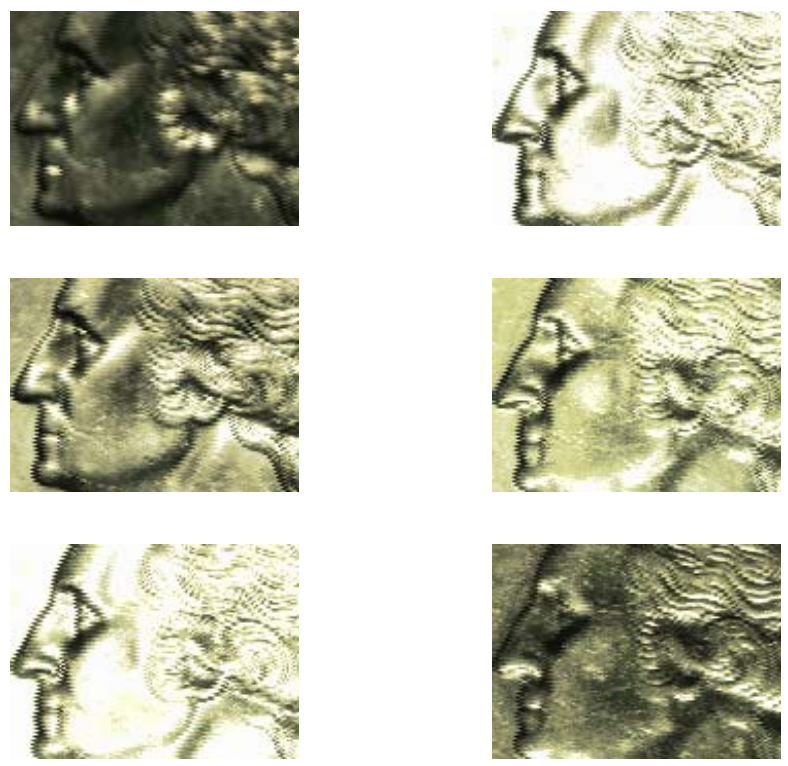

Figure 10. Texture images for the coin. Let the viewing direction be denoted $\gamma_{v}, \beta_{v}$. Let the illumination direction be denoted $\gamma_{l}, \beta_{l}$. Left column is for the same viewing direction, i.e., $\gamma_{v}=12^{\circ}, \beta_{v}=90^{\circ}$. From the top row to the bottom row in this column, the illumination direction changes from $\gamma_{l}=9.3^{\circ}, \beta_{l}=90^{\circ}$, to $\gamma_{l}=0^{\circ}$, to $\gamma_{l}=15.26^{\circ}, \beta_{l}=-90^{\circ}$. Right column is for the same illumination direction, i.e., $\gamma_{l}=11.1^{\circ}, \beta_{l}=-90^{\circ}$. From the top row to the bottom row in this column, the viewing direction changes from $\gamma_{v}=15^{\circ}, \beta_{v}=90^{\circ}$, to $\gamma_{v}=0^{\circ}$, to $\gamma_{v}=19^{\circ}, \beta_{v}=-90^{\circ}$.

IEEE Transactions on Pattern Analysis and Machine Intelligence, 3(6):661-669, 1981.

[8] J. J. Koenderink and A. J. van Doorn. Illuminance texture due to surface mesostructure. Journal of the Optical Society of America A, 13:452-463, 1996.

[9] J. J. Koenderink, A. J. van Doorn, K. J. Dana, and S. K. Nayar. Bidirectional reflection distribution function of thoroughly pitted surfaces. International Journal of Computer Vision, 31(2-3):129-144, 1999.

[10] T. Leung and J. Malik. Representing and recognizing the visual appearance of materials using threedimensional textons. International Journal of Computer Vision, 43(1):29-44, June 2001.

[11] M. Levoy, K. Pulli, B. Curless, S. Rusinkiewicz, D. Koller, L. Pereira, M. Ginzton, S. Anderson, J. Davis, J. Ginsberg, J. Shade, and D. Fulk. The digital michelangelo project: $3 \mathrm{~d}$ scanning of large statues. ACM SIGGRAPH, pages 131-144, 2000.
[12] X. Liu, Y. Yu, and H.Y. Shum. Synthesizing bidirectional texture functions for real world surfaces. $A C M$ SIGGRAPH, pages 97-106, 2001.

[13] W. Matusik, H. Pfister, A. Ngan, P. Beardsley, R. Ziegler, and L. McMillan. Image-based 3d photography using opacity hulls. ACM SIGGRAPH, pages 427-437, 2002.

[14] S. K. Nayar, K. Ikeuchi, and T. Kanade. Determining shape and reflectance of hybrid surfaces by photometric sampling. IEEE Trans. On Robotics and Automation, 6(4):418-431, 1990.

[15] S. C. Pont and J. J. Koenderink. Bidirectional texture contrast function. Proceedings of the European Conference on Computer Vision, pages 808-822, 2002.

[16] S. Rusinkiewicz, O. Hall-Holt, and M. Levoy. Realtime $3 \mathrm{~d}$ model acquisition. ACM SIGGRAPH, pages 438-446, 2002.

[17] M. Varma and A. Zisserman. Classifying images of materials. Proceedings of the European Conference on Computer Vision, pages 255-271, 2002.

[18] Y. Z. Yu, A. Ferencz, and J. Malik. Extracting objects from range and radiance images. IEEE Transactions on Visualization and Computer Graphics, 7(4):351364, 2001.

[19] A. Zalesny and L. Van Gool. Multiview texture models. Proceedings of the IEEE Conference on Computer Vision and Pattern Recognition, 1:615-622, December 2001. 